\title{
Variación espacio-temporal de la regeneración natural arbórea y arbustiva de bosque de alta montaña en etapa inicial de desarrollo en el sur de Brasil
}

\author{
Spatial-temporal variation of the natural regeneration of an early-successional \\ upper-montane tree-shrub community in Southern Brazil
}

\author{
Janaina Gabriela Larsen a, Edilaine Duarte a, Jaqueline Beatriz Brixner Dreyer a, \\ Juliana Pizutti Dallabrida a, Luran Monteiro Muzeka a, Guilherme Neto dos Santos a,

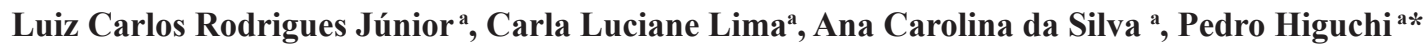 \\ *Autor de correspondencia: ${ }^{a}$ Universidad del Estado de Santa Catarina, Departamento de Ingeniería Forestal, \\ Av. Luiz de Camões, 2090, Lages, Brasil, tel.: 55-49-32899308, higuchip@gmail.com
}

\begin{abstract}
SUMMARY
We aimed at analyzing the dynamics of spatial-temporal variations of the early-successional wood and sub-woody components in an upper-montane community, in the municipality of Urubici, Southern Brazil. Within a 20x100 m transect, all regenerative individuals, equal to or over $20 \mathrm{~cm}$ high and with diameter at breast height smaller than $5 \mathrm{~cm}$, were identified, measured, tagged and spatially located (x, y coordinates). One and two years after the first survey, all sampled individuals were counted again, registering recruits and dead individuals. Demographic rates by size class and time interval (2014-2015 and 2015-2016), number of individuals of the most abundant species and spatial distribution of dead and recruits were determined. We observed the increment of number of individuals and a greater participation of early-successional species. Smaller size classes and second time interval had superior values of mortality and recruitment rates. For both time intervals, recruits presented a clustered spatial pattern. Dead individuals showed, predominantly, a random spatial pattern. Results indicated structural instability, demographic rates variation regarding size classes and time interval, and distinct spatial pattern for dead and recruited individuals. We conclude that regeneration of the study area is still at an early stage of succession, presenting itself as a complex process, expressed by different spatial patterns of mortality and recruitment.
\end{abstract}

Key words: dynamics, spatial distribution, vegetation succession.

\section{RESUMEN}

El presente estudio tuvo como objetivo analizar la variación espacio-temporal de la dinámica del componente leñoso y sub-leñoso regenerado en una comunidad de alta montaña en fase inicial de la sucesión del bosque en Urubici (Santa Catarina, sur de Brasil). Sobre un transecto de 20x100 m, se identificaron las especies, se midieron y marcaron los individuos regenerados con altura $\geq 20 \mathrm{~cm}$ y diámetro a la altura del pecho $<5 \mathrm{~cm}$, además se obtuvieron las coordenadas espaciales ( $\mathrm{x}, \mathrm{y})$. Uno y dos años después de la primera evaluación, los individuos muestreados fueron recontados, aquellos que alcanzaron la categoría de inclusión fueron reclutados y se consideraron los muertos. Se determinaron las tasas demográficas por clases de tamaño y período de estudio (2014-2015 y 20152016), la variación del número de individuos de las especies más abundantes y la distribución espacial de muertos y reclutados. Hubo aumento del número de individuos. Las tasas de mortalidad y reclutamiento fueron mayores en las clases de tamaño más pequeñas y en el segundo período de evaluación. Los individuos reclutados tendieron a presentar atracción espacial en ambos períodos y los muertos presentaron patrón aleatorio. Se demostró incremento en abundancia, variación de las tasas de dinámica según clase de tamaño y período de estudio, como patrones distintos de distribución espacial de individuos muertos y reclutados. Se concluyó que la regeneración natural del área de estudio aún se encuentra en fase inicial, presentándose como un proceso de alta complejidad, expresado por diferentes patrones espaciales de mortalidad y reclutamiento.

Palabras clave: dinámica, distribución espacial, sucesión vegetal.

\section{INTRODUCCIÓN}

El bosque con araucaria (Araucaria angustifolia (Bertol.) Kuntze) de alta montaña está localizado en el sur de Brasil en regiones arriba de $1.000 \mathrm{~m}$ de altitud, formando un mosaico de campos y bosques (IBGE 2012). En estas regiones predominan bajas temperaturas, con heladas fre- cuentes, caracterizando un ambiente limitante para el establecimiento de especies arbóreas (Higuchi et al. 2012). Por eso, estos bosques se caracterizan por la reducida riqueza de especies arbóreas adaptadas a las condiciones ambientales peculiares y por el elevado endemismo (Marcon et al. 2014). Además de ser ecosistemas singulares por su composición florística peculiar, en el sur de Brasil estos bos- 
ques cumplen importantes funciones ambientales, como, por ejemplo, protegiendo los manantiales de los principales ríos de la región (Soboleski et al. 2017). Sin embargo, los bosques con araucaria de alta montaña, así como los demás situados en sitios de altitud más alta, están siendo amenazados por acciones antropogénicas y cambios climáticos, especialmente los relacionados al calentamiento global (Ponce-Reyes et al 2013).

Estos bosques forman parte del bioma Mata Atlántica, considerado un hotspot mundial para la conservación (Myers et al. 2000), y están en peligro debido al cambio de uso del suelo por actividades humanas, como la ganadería (Sevegnani et al. 2012). De este modo, una mayor comprensión acerca del proceso de sucesión vegetal en áreas con diferentes historias de perturbación es fundamental, pues puede generar datos que detecten la necesidad de acciones facilitadoras de la regeneración natural, así como subsidios para la recuperación de otras áreas degradadas similares.

La sucesión vegetal en bosques secundarios es un proceso complejo, cuya velocidad se relaciona con la resiliencia del área perturbado, con variaciones que ocurren a lo largo del espacio y del tiempo (Van Breugel et al. 2006, Arroyo-Rodríguez et al. 2017). Los cambios en la vegetación arbórea se dan en función de la dinámica del bosque, que pueden ser expresados por las tasas demográficas de mortalidad, reclutamiento y crecimiento de los árboles (Gross et al. 2017). Hay varios factores ecológicos determinantes en este proceso, como aquellos de naturaleza estocástica y determinística, que determinan las características estructurales y espaciales de la vegetación (Silveira et al. 2017). El agrupamiento espacial de los individuos muertos y reclutados sugiere, respectivamente, la influencia de factores dependientes de la densidad (por ejemplo, competencia inter e intraespecífica; Larson et al. 2016) y de nucleación (por ejemplo, perchas naturales, micrositios favorables a la regeneración; Korndörfer et al. 2015). Por otro lado, patrones completamente aleatorios pueden surgir de la influencia de factores estocásticos (Salami et al. 2014), como, por ejemplo, eventos climáticos extremos en la mortalidad de individuos. De esta forma, las alteraciones en una comunidad de plantas a lo largo del tiempo, así como los patrones espaciales de los eventos demográficos, pueden proporcionar informaciones sobre la orientación del proceso de sucesión.

En vista de lo expuesto, el objetivo es analizar la variación espacio-temporal de la dinámica del componente arbóreo y arbustivo de la regeneración en una comunidad de alta montaña en fase inicial de sucesión, en sur de Brasil. Las hipótesis son que i) el proceso de sucesión se caracteriza por la ganancia en número de individuos; y ii) la mortalidad y el reclutamiento ocurren de forma espacial agregada, reflejando aspectos relacionados a la competición inter e intra específica de plantas y nucleación. Para ello, se analizan las tasas demográficas, así como la estructura de la comunidad y de las principales poblaciones, lo que suministrará informaciones acerca de la organización de la comunidad.

\section{MÉTODOS}

Este trabajo fue desarrollado en un área abierta deforestada en el pasado (coordenadas: $28^{\circ} 04^{\prime} 55,82^{\prime}$ ' S y $49^{\circ} 30^{\prime} 42,37^{\prime \prime} \mathrm{W}$ ), pero que hace 10 años está protegida, desde el momento en que fue agregada al Parque Nacional de São Joaquim, tramo ubicado en el "Morro da Igreja", municipio de Urubici, Santa Catarina, Brasil (figura 1). El Parque, creado en el año 1961, tuvo, a lo largo del tiempo, áreas expropiadas y adjuntas, que actualmente se encuentran protegidas y en proceso de sucesión vegetal. Antes de la deforestación, el área de estudio era de bosque ombrofilo mixto de alta montaña, así como las áreas adyacentes (fragmentos remanentes). A su vez, tiene una altitud media de 1.356 m s.n.m., y el clima de la región según la clasificación climática de Köppen es el Cfb (templado húmedo con veranos moderados). De acuerdo con el WorldClim (Hijmans et al. 2005), la precipitación media anual es de $1.753 \mathrm{~mm}$ y la temperatura media anual es de $12,7^{\circ} \mathrm{C}$ para el período desde el año 1950 hasta 2000. Los suelos se clasifican como litosoles y cambisoles.

En el primer inventario (Dallabrida et al. 2017) en 2014, fue instalado un transecto de 20x100 m, dividida en 20 subparcelas de $10 \times 10 \mathrm{~m}$. Sobre toda la superficie del transecto, los autores muestrearon los individuos leñosos y sub-leñosos iguales o mayores a $20 \mathrm{~cm}$ de altura, y aquellos con diámetro a la altura del pecho medido a $1,30 \mathrm{~m}$ del suelo inferior a $5 \mathrm{~cm}$. Todos los individuos fueron marcados, identificados y sus alturas medidas. Las coordenadas espaciales $(\mathrm{x}, \mathrm{y})$ se han obtenido con un medidor de distancia ultrasónico DME 201 Haglöf. En 2015 y en 2016, uno y dos años tras el primer inventario, fueron inventariados nuevamente los individuos, siendo los sobrevivientes medidos de nuevo y los muertos contabilizados. Los individuos que alcanzaron la altura de inclusión $(20 \mathrm{~cm})$ han sido considerados como reclutamiento, siendo, por lo tanto, marcados con placas de aluminio, identificados, medidos y obtenidas sus coordenadas $\mathrm{x}$ e $\mathrm{y}$.

Las tasas de mortalidad (M, en \%.año ${ }^{-1}$ ) y de reclutamiento $\left(\mathrm{R}\right.$, en $\%$.año $\left.\mathrm{o}^{-1}\right)$ han sido calculadas, por clase de altura, para toda la comunidad, con base a las fórmulas utilizadas por Korning y Balslev (1994) y Sheil et al. (2000) [1], [2]:

$$
\begin{gathered}
M=\left[1-\left(\frac{\mathrm{N} 1-\mathrm{Nmue}}{\mathrm{N} 1}\right)^{\frac{1}{\mathrm{t}}}\right] \times 100 \\
\mathrm{R}=\left[1-\left(1-\frac{\mathrm{Nrec}}{\mathrm{N} 2}\right)^{\frac{1}{\mathrm{t}}}\right] \times 100
\end{gathered}
$$

Donde, N1 = número inicial de individuos; Nmue = número de individuos muertos; $\mathrm{t}=$ tiempo entre los inventarios; $\mathrm{Nrec}=$ número de individuos reclutados; $\mathrm{N} 2$ = número final de individuos. 


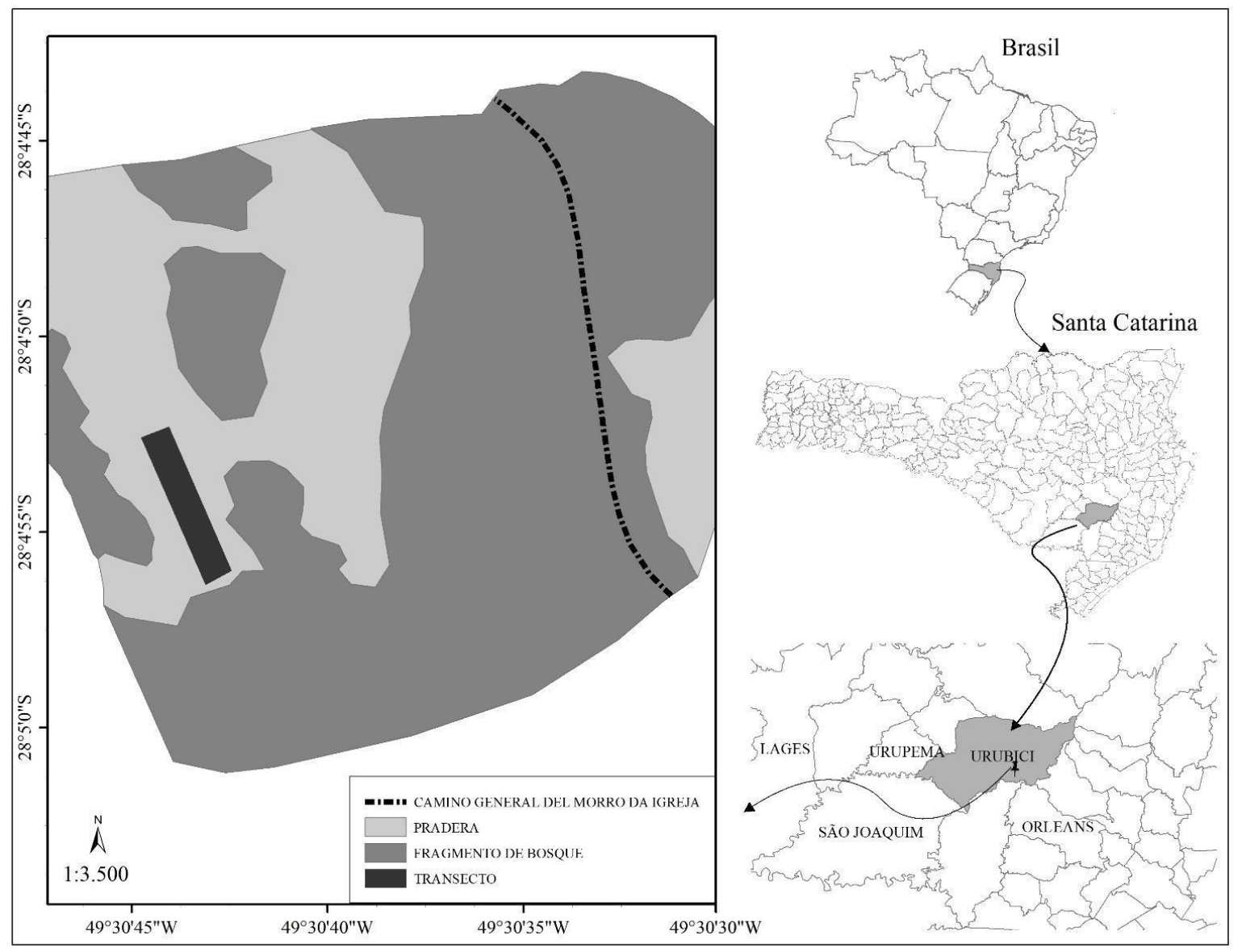

Figura 1. Localización del área de estudio en el Parque Nacional de São Joaquim, município de Urubici, Santa Catarina, Brasil. Study area location in São Joaquim National Park, in the municipality of Urubici, Santa Catarina, Brazil.

La representación gráfica de los resultados se dio por histogramas, en función de las clases de altura y para cada período de estudio. El número de clases $(\mathrm{k})$ fue calculado mediante la regla de Sturges (Sturgers, 1926) [3] y la amplitud de clases (A) dada por la fórmula [4]:

$$
\begin{gathered}
\mathrm{k}=1+3,33 \times \log (\mathrm{n}) \\
\mathrm{A}=(\text { máximo valor }- \text { mínimo valor }) / \mathrm{k}
\end{gathered}
$$

Donde, $\mathrm{n}=$ número de individuos regenerados muestreados.

El patrón espacial entre los individuos muertos y reclutados se analizó usando la función L univariada, con una corrección del efecto de borde. Esta función es una transformación propuesta por Besag (1977) de la función K (Ripley 1977) [5]:

$$
\mathrm{L}(\mathrm{r})=\sqrt{ }(\mathrm{K}(\mathrm{r}) / \mathrm{pi})
$$

Donde, $\mathrm{r}=$ son los radios crecientes alrededor a cada proceso puntual; $\mathrm{K}=$ los valores de la función $\mathrm{K}$, para un determinado valor de $\mathrm{r}$, con el propósito de visualización gráfica.

Las significaciones de los patrones espaciales observados fueron probadas por medio de la construcción de intervalos de confianza de aleatoriedad espacial completa, a partir de 999 permutaciones, lo que representa un alfa de 0,01 . A través de la prueba de Chi-cuadrado y de la estadística de razón de verosimilitud $(P<0,05)$, se analizó la existencia de patrones espaciales de primer orden, ya que pueden influenciar en las relaciones espaciales entre los individuos muertos y los reclutados. Para ello, los patrones espaciales observados fueron considerados como variables dependientes en función de las coordenadas cartesianas a lo largo de la mayor longitud de los transectos muestrales. Este procedimiento fue realizado por medio de las funciones en R (R Development Core Team 2017) ppm y anova. ppm, del paquete spatstat v. 1.57-1 (Baddeley y Turner 2005). En el caso de un patrón espacial no estacionario, la función $\mathrm{L}$ se determinó según la metodología descrita por Baddeley et al. (2000), utilizando la función Linhom, con el paquete spatstat. Con el propósito de visualización gráfica de los eventos de dinámica, fueron trazadas las dis- 
tribuciones espaciales de los individuos muertos y reclutados, y luego se determinó la probabilidad de ocurrencia por el método de estimación de la densidad del grano, con la función density, del mismo paquete $\mathrm{R}$.

\section{RESULTADOS}

Durante el período de estudio se observaron valores crecientes de abundancia de los individuos en la comunidad: 696, 727 y 790 individuos regenerados en los años 2014, 2015 y 2016, respectivamente (cuadro 1). Por lo tanto, las entradas, mediante el reclutamiento, superaran a las salidas (mortalidad).
Sin embargo, las tasas de mortalidad (figura 2A) o de reclutamiento (figura $2 \mathrm{~B}$ ) presentaron variaciones en función de la clase de tamaño, con la presencia de mayores valores para individuos de menor tamaño, de manera que el patrón se asemejó a una distribución de frecuencia exponencial negativa ("J invertida") en ambos períodos de evaluación. En general, los mayores valores de las tasas también fueron encontrados en el período 2015-2016 que en el período anterior.

La mortalidad presentó, principalmente, elevados valores para las especies Senecio brasiliensis y Croton cf. patrum, para ambos períodos de evaluación. Para el reclutamiento, las especies más representativas fueron $C$. cf.

Cuadro 1. Abundancia de especies leñosas y sub-leñosas en un área de sucesión inicial en el Parque Nacional de São Joaquim, sur de Brasil.

Woody species abundance in an early-successional area, in São Joaquim National Park, southern Brazil.

\begin{tabular}{|c|c|c|c|}
\hline Especies leñosas y sub-leñosas & 2014 & 2015 & 2016 \\
\hline Baccharis uncinella DC. & 188 & 184 & 181 \\
\hline Campovassouria cruciata (Vell.) R.M.King et H.Rob. & 143 & 172 & 197 \\
\hline Senecio brasiliensis (Spreng.) Less. & 105 & 96 & 28 \\
\hline Croton cf. patrum L.B.Sm. et Downs & 92 & 62 & 50 \\
\hline Berberis laurina Thunb. & 27 & 30 & 34 \\
\hline Solanum paranense Dusén & 25 & 47 & 55 \\
\hline Myrceugenia euosma (O.Berg) D. Legrand & 17 & 17 & 17 \\
\hline Mimosa scabrella Benth. & 11 & 8 & 5 \\
\hline Baccharis microdonta DC. & 10 & 27 & 44 \\
\hline Cinnamoтum amoenum (Nees) Kosterm. & 10 & 10 & 9 \\
\hline Myrceugenia glaucescens (Cambess.) D.Legrand et Kausel & 10 & 10 & 10 \\
\hline Myrceugenia regnelliana (O.Berg) D. Legrand et Kausel & 10 & 10 & 10 \\
\hline Vernonanthura montevidensis (Spreng.) H.Rob. & 10 & 10 & 9 \\
\hline Myrsine coriacea (Sw.) R.Br. & 8 & 11 & 31 \\
\hline Araucaria angustifolia (Bertol.) Kuntze. & 7 & 7 & 7 \\
\hline Leandra sp. 1 & 5 & 7 & 54 \\
\hline Escallonia bifida Link et Otto & 4 & 4 & 4 \\
\hline Drimys angustifolia Miers & 3 & 3 & 9 \\
\hline Myrceugenia oxysepala (Burret) D.Legrand et Kausel. & 3 & 4 & 4 \\
\hline Buddleia reitzii E. M. Norman et L. B. Sm. & 2 & 2 & 2 \\
\hline Leandra sp. 2 & 2 & 2 & 14 \\
\hline Clethra uleana Sleumer & 1 & 1 & 1 \\
\hline Mimosa pilulifera Benth. & 1 & 1 & 1 \\
\hline Siphoneugena reitzii D. Legrand & 1 & 1 & 1 \\
\hline Solanum pseudocapsicum L. & 1 & 0 & 9 \\
\hline Maytenus boaria Molina & 0 & 0 & 2 \\
\hline Myrceugenia myrcioides (Cambess.) O. Berg & 0 & 1 & 2 \\
\hline Total & 696 & 727 & 790 \\
\hline
\end{tabular}


patrum y S. brasiliensis, en el primer período de evaluación (2014-2015), y Campovassouria cruciata y S. brasiliensis, en el segundo período (2015-2016). Este patrón puede ser explicado, en parte, por la alta densidad de esas especies en la comunidad (cuadro 1), ya que todas son iniciales de sucesión.

Campovassouria cruciata fue la de mayor densidad en 2016, tras superar Baccharis uncinella, que era la especie de mayor abundancia en los años 2014 y 2015 y obtuvo

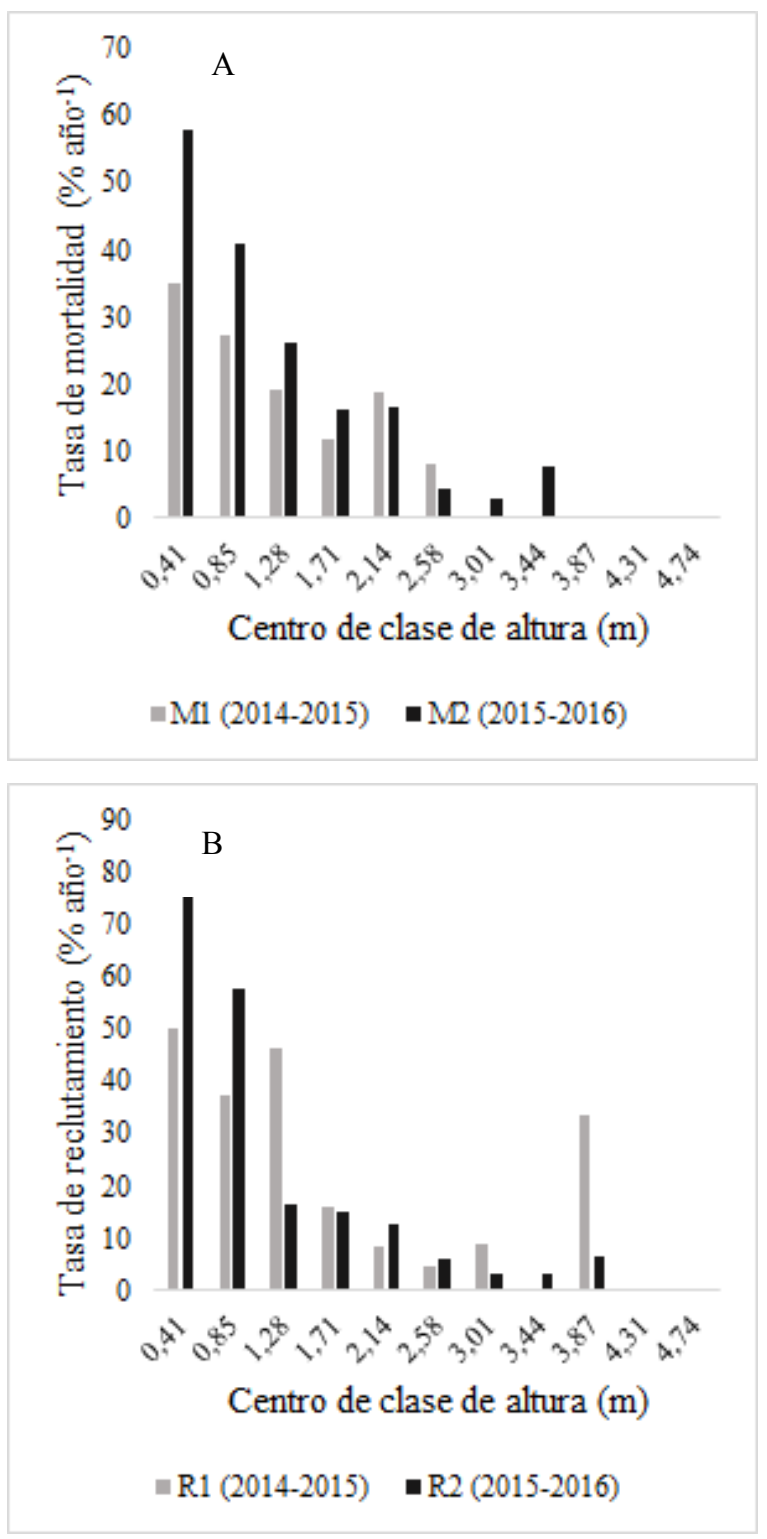

Figura 2. A) Tasas de mortalidad (M) y B) reclutamiento (R) por clase de altura, en los períodos 2014-2015 y 2015-2016, en un área de sucesión inicial en el Parque Nacional de São Joaquim, sur de Brasil.

A) Mortality (M) and B) recruitment (R) rates by size classes, during 2014-2015 and 2015-2016 intervals, in an early-successional area, in São Joaquim National Park, southern Brazil. pequeña reducción de su población durante los períodos de evaluación. Entre las cinco especies de mayor densidad, $S$. brasiliensis y $C$. cf. patrum se redujeron de forma expresiva y Berberis laurina tuvo un aumento sustancial de su representatividad.

Considerando la estructura espacial, para la mortalidad observada en 2015 (figura 3A), se verificó por medio de ANOVA para modelos de procesos puntuales espaciales ajustados (anova.ppm) una tendencia de distribución espacial de primer orden (patrón espacial no estacionario) ( $D e-$ viance $=5,37 ; P<0,05)$, de modo que, a lo largo del eje-x, se aumenta el número de muertos, lo que indica un proceso espacial no homogéneo. Para el reclutamiento (figura 3B), de acuerdo con la misma prueba estadística, no hubo tendencia de primer orden (Deviance $=0,04 ; P>0,05)$, o sea, el establecimiento de reclutados se produjo de forma homogénea a lo largo de todo lo transecto. Al analizar el patrón espacial de segundo orden (relación espacial entre los individuos) (figura 4), se observa que, mientras los muertos se dieron sin relación de dependencia espacial, señalando completa aleatoriedad entre ellos (figura 4A), los reclutados presentaron asociación espacial predominantemente positiva, indicando atracción, hasta un radio de $5 \mathrm{~m}$ (figura 4B). Este patrón demuestra que el establecimiento de individuos jóvenes, que ocurrió principalmente en la clase más pequeña de altura (figura 2B), se dio de forma adensada, formando "nubes" de reclutamiento con un radio de hasta $5 \mathrm{~m}$.

Para el año de 2016, la estructuración espacial de primer orden para los eventos demográficos de mortalidad y reclutamiento (figura 3C, D) fueron iguales a los observados en el año anterior, con tendencia de distribución no homogénea para los muertos y una distribución homogénea para los reclutados. La ocurrencia de los muertos, en este último período de inventario, presentó un patrón espacial de segundo orden más complejo, de manera aleatoria entre sí en un radio hasta $1 \mathrm{~m}$ (figura 4C), y de repulsión espacial entre 1 y $5 \mathrm{~m}$. Por otro lado, los reclutados se presentaron de forma agregada en un radio de hasta $3 \mathrm{~m}$ y, desde ese valor hasta $5 \mathrm{~m}$, de forma aleatoria (figura 4D), indicando la formación de nubes de reclutamiento de hasta $3 \mathrm{~m}$ de radio.

\section{DISCUSIÓN}

Los resultados obtenidos permiten verificar que el área presenta aumento del número de individuos a lo largo del tiempo, y por la elevada participación de especies iniciales de sucesión. De esta forma, considerando el patrón de dinámica observado y la elevada representatividad de especies pioneras, es posible inferir que, aproximadamente 10 años después en el área protegida, la vegetación aún se encuentra en estado de sucesión inicial. Áreas en estado inicial de sucesión vegetal presentan mayor reclutamiento que mortalidad, pues el bosque está en etapa de estructuración, con espacios libres para la llegada de plántulas, resultando en el aumento de la densidad (Sanquetta et al. 2003). Evidencias (Guariguata y Ostertag 2001, Van Breu- 
gel et al. 2006) demuestran que la velocidad de este proceso es más rápida en la fase inicial, en las primeras décadas, que en la tardía. Asimismo, áreas de selva atlántica en la región Sur de Brasil pueden llevar de uno a tres siglos para alcanzar la madurez (Liebsch et al. 2008). Además de las variaciones temporales, variaciones espaciales ocurren en función de la influencia de varios factores de dimensión local, como el régimen de perturbación, la calidad del ambiente edáfico entre otros factores ambientales, como la competencia y la distancia a la fuente de propágulos (Guariguata y Ostertag 2001, Arroyo-Rodríguez et al. 2017). Solamente a partir de una evaluación con mayor intervalo

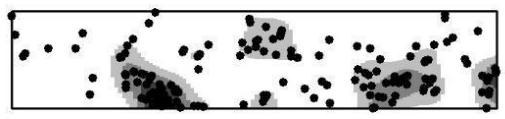

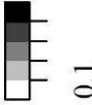

A
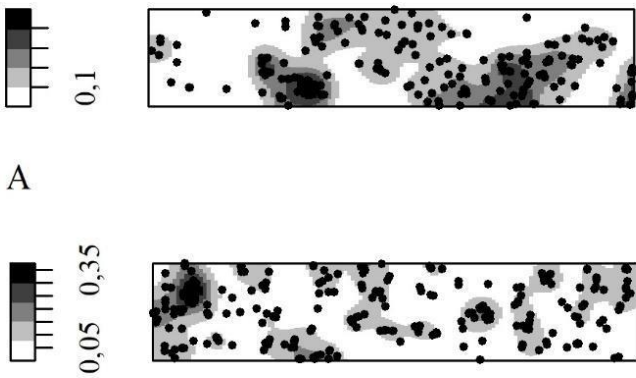

B

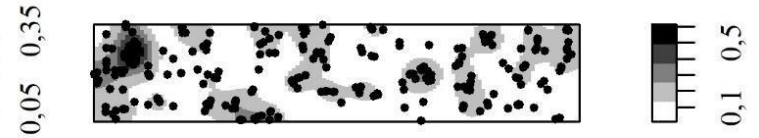

D

Figura 3. Distribución espacial de los individuos leñosos y sub-leñosos muertos (A, C) y reclutados (B, D) en 2015 (A, B) y 2016 (C, D), en un área de sucesión inicial en el Parque Nacional de São Joaquim, sur de Brasil. La escala de grises indica que las regiones más oscuras presentan mayor probabilidad de ocurrencia del evento.

Spatial distribution of dead (A, C) and recruits (B, D) woody and sub-woody individuals in 2015 (A, B) and 2016 (C, D), in an early-successional area, in São Joaquim National Park, southern Brazil. The grayscale gradient indicates a higher probability of events occurrence for darker areas.
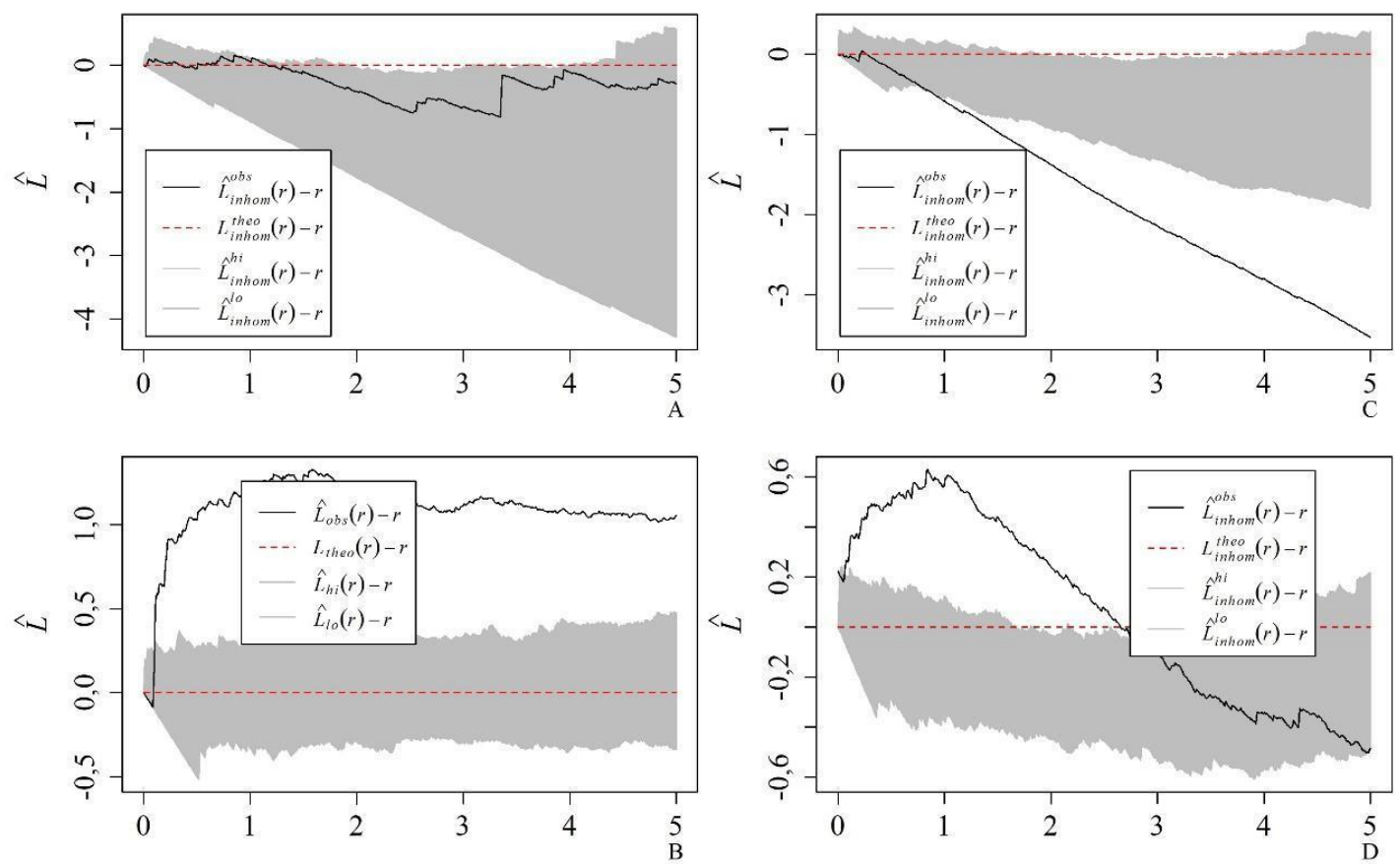

Figura 4. Función L para los individuos leñosos y sub-leñosos muertos (A, C) y reclutados (B, D) en 2015 (A, B) y 2016 (C, D), en función de un radio (r) de hasta $5 \mathrm{~m}$. La línea negra continua representa el valor de L observado. El intervalo gris representa una relación de aleatoriedad espacial completa. Valores de L por encima del intervalo gris señalan asociación espacial positiva (atracción). Valores de L por debajo del intervalo gris indican asociación espacial negativa (repulsión).

$L$ function of dead (A, C) and recruits (B, D) woody and sub-woody individuals in 2015 (A, B) and 2016 (C, D), as a function of a radius up to $5 \mathrm{~m}$ (r). Dark line represents $L$ observed value. Gray envelope indicates a complete randomly spatial relation. $L$ values above gray envelope indicates positive spatial association (attraction). $L$ values below gray envelope indicates negative spatial association (repulsion). 
de tiempo sería posible detectar la sustitución por especies tardías. Sin embargo, las evaluaciones en períodos de corto plazo son de gran importancia, porque pueden captar los cambios del inicio de la sucesión. Así, es evidente que la sucesión vegetal es un proceso de alta complejidad, que puede presentar variaciones de velocidad a lo largo del tiempo y en el espacio.

En el área del estudio, aunque esté protegida actualmente del impacto antropogénico, disturbios naturales, como heladas frecuentes y nieve en invierno, tienen el potencial de impactar en las poblaciones existentes, causando mortalidad. Se observó, en el área, que una intensa helada en el invierno de 2016 generó gran mortalidad de Senecio brasiliensis, lo que permite decir que esta especie es poco tolerante a eventuales congelamientos. De hecho, la localidad de estudio está sujeta a las condiciones microclimáticas extremas, tales como temperatura $-15{ }^{\circ} \mathrm{C}$, viento con hasta $100 \mathrm{~km} / \mathrm{h}$ y congelación de la superficie del suelo durante varios días en el invierno (Falkenberg 2003) Luego, condiciones como estas podrían ocasionar desórdenes de mortalidad más severas en la comunidad, haciendo que el proceso de sucesión sea más lento. Este es un patrón plausible, considerando que el impacto de eventos climáticos extremos sobre la vegetación, como aquellos relacionados con heladas severas, han sido reportados en la literatura (Nascimento et al. 1999, Demarchi y Piroli 2015). De esta forma, a pesar de que otros factores no fueron evaluados, se puede inferir que las condiciones climáticas más severas en el período de invierno de 2016 en la región del Parque Nacional de São Joaquim sea un posible candidato para explicar la mortalidad más pronunciada observado en los años 2015-2016.

El mayor número de individuos muertos en las categorías más jóvenes de la vegetación es un patrón esperado, pues, por lo general, individuos más jóvenes son más susceptibles a las variaciones bióticas y abióticas del medio (Janzen 1970, Jabot et al. 2008). Factores ambientales limitantes podrían actuar de forma más intensa en individuos más jóvenes (Jabot et al. 2008) o se puede relacionar la mayor mortalidad a la competencia, herbivoría o depredación, más común en este estrato de vegetación (Janzen 1970). Sin embargo, además de la mayor mortalidad en el estrato de menor altura, el reclutamiento también fue superior en ese estrato, y ha superado la mortalidad. Este era un resultado esperado, considerando que en la menor clase se encuentra la mayoría de los reclutados, o sea, aquellos que alcanzaron el nivel mínimo de inclusión.

Son muchos los procesos ecológicos que pueden determinar los patrones espaciales de primer y segundo orden para los eventos demográficos de mortalidad y reclutamiento en áreas en estado inicial de sucesión. La distribución homogénea de reclutados y no homogénea de muertos, que refleja en el patrón espacial de primer orden, puede ser el resultado de, respectivamente, una dispersión de propágulos espacialmente limitada y condiciones ambientales no limitantes (Nathan y Muller-Landau 2000).
La existencia de correlación positiva entre los reclutados en una escala espacial pequeña $(<5 \mathrm{~m})$ sugiere la influencia de componentes de nucleación en el paisaje. De hecho, para el Sur de Brasil, árboles adultos de Araucaria angustifolia son importantes elementos facilitadores de regeneración natural de especies arbóreas, funcionando como perchas naturales para las aves dispersoras de semillas y mejorando las condiciones ambientales (Korndörfer et al. 2015). En la misma zona geográfica, las rocas, los helechos arborescentes muertos y troncos caídos son componentes de nucleación que influyen en la distribución de especies zoocoras (Dallabrida et al. 2017). En áreas de fase inicial de sucesión, se espera que la mortalidad de individuos jóvenes sea un proceso dependiente de la densidad, relacionado con la competencia inter e intraespecífica, reflejando, de esa manera, un patrón de agrupación espacial (Larson et al. 2015). Sin embargo, este no fue el patrón encontrado, lo que sugiere, principalmente, la relevancia de factores estocásticos en el área evaluada, como, por ejemplo, fenómenos climáticos extremos, como heladas severas que ocurren en la región. Además, entre 1 y $5 \mathrm{~m}$ de distancia de cada individuo muerto, solo para el segundo año de evaluación, el patrón de repulsión indica la tendencia de un área vacía, sin la ocurrencia de otra mortalidad.

\section{CONCLUSIONES}

El patrón de dinámica de la comunidad indicó inestabilidad estructural e inicio de proceso sucesión, caracterizado por un aumento del número de individuos y por la participación de especies iniciales de sucesión como las más representativas de la regeneración natural. Además, la mortalidad y el reclutamiento fueron mayores en las menores clases de altura, como también en el segundo período de evaluación. La estructuración espacial de los eventos demográficos ha permitido concluir que, mientras el reclutamiento fue dependiente de la influencia de elementos que favorecieron la densificación de individuos, la mortalidad ocurrió de modo predominantemente aleatoria. Sin embargo, se destaca que los resultados y patrones encontrados no deben ser generalizados, teniendo en vista que el muestreo contempló solo condiciones locales del área de estudio, que no son representativas de la fitofisionomía evaluada.

\section{AGRADECIMIENTOS}

A FAPESC (Brasil), por el apoyo financiero mediante el Edicto PAP/FAPESC/UDESC. Al CNPq (Brasil), por el apoyo financiero del proyecto por medio del Edicto MCTI/ CNPQ/Universal 14/2014 y por la concesión de Beca de Investigación a la primera autora del estudio y de Becas de Productividad al noveno y décimo autores. Al Instituto Chico Mendes de Conservação da Biodiversidade y al personal del Parque Nacional de São Joaquim, por el permiso de ejecución del trabajo. 


\section{REFERENCIAS}

Arroyo-Rodríguez V, FP Melo, M Martínez-Ramos, F Bongers, RL Chazdon, JA Meave, N Norden, BA Santos, IR Leal, M Tabarelli. 2017. Multiple successional pathways in human-modified tropical landscapes: new insights from forest succession, forest fragmentation and landscape ecology research. Biological Reviews 92(1): 326-340. DOI: 10.1111/ brv. 12231

Baddeley A, J Moller, R Waagepetersen. 2000. Non- and semiparametric estimation of interaction in inhomogeneous point patterns. Statistica Neerlandica 54: 329-350. DOI: $\underline{10.1111 / 1467-9574.00144}$

Baddeley A, R Turner. 2005. spatstat: An R Package for Analyzing Spatial Point Patterns. Journal of Statistical Software 12(6): 1-42. DOI: $10.18637 /$ jss.v012.i06

Besag J. 1977. Discussion of Dr Ripley's paper. Journal of the Royal Statistical Society, Series B 39: 193-195.

Dallabrida JP, AC Silva, P Higuchi, K Souza, R Loebens, LC Rodrigues-Junior, VF Soboleski, JG Larsen, FF Walter, RV Kilca. 2017. Elementos nucleadores da paisagem influenciam a dispersão zoocórica em áreas campestres alto-montanas. Rodriguesia 68(2): 325-335. DOI: $10.1590 / 2175-$ 7860201768204

Demarchi JC, EL Piroli. 2015. Análise dos efeitos de geadas na cobertura vegetal do município de Cândido Mota - SP, Brasil, por meio de Índices de Vegetação. Boletim Goiano de Geografia 35(3): 554-574. DOI: 10.5216/bgg.v35i3.38843

Falkenberg DB. 2003. Matinhas nebulares e vegetação rupícola dos Aparados da Serra Geral (SC/RS), Sul do Brasil. Tesis Magíster en Biologia Vegetal. Campinas, Brazil. Universidade Estadual de Campinas. 558 p. http://repositorio.unicamp.br/jspui/handle/REPOSIP/315046

Guariguata MR, R Ostertag. 2001. Neotropical secondary forest succession: changes in structural and functional characteristics. Forest Ecology and Management 148(1): 185-206. DOI: $10.1016 / \mathrm{S} 0378-1127(00) 00535-1$

Gross A, AC Silva, AP Cruz, RV Kilca, AS Nunes, E Duarte, MB Vefago, GN Santos, CL Lima, B Salami, P Higuchi. 2017. Fragmentation as a key driver of tree community dynamics in mixed subtropical evergreen forests in Southern Brazil. Forest Ecology and Management 411: 20-26. DOI: 10.1016/j.foreco.2018.01.013

Higuchi P, AC Silva, TS Ferreira, ST Souza, JP Gomes, KM Silva, KF Santos. 2012. Floristic composition and phytogeography of the tree component of Araucaria Forest fragments in southern Brazil. Brazilian Journal of Botany 35(2): 145157. DOI: $10.1590 / \mathrm{S} 0100-84042012000200004$

Hijmans RJ, SE Cameron, JL Parra, PG Jones, A Jarvis. 2005. Very high resolution interpolated climate surfaces for global land areas. International Journal of Climatology 25(15): 1965-1978. DOI: $\underline{10.1002 / j o c .1276}$

IBGE (Instituto Brasileiro de Geografia e Estatística, BR). 2012. Manual Técnico da Vegetação Brasileira. Rio de Janeiro, Brazil. Instituto Brasileiro de Geografia e Estatística. 271 p.

Jabot F, RS Etienne, J Chave. 2008. Reconciling neutral community models and environmental filtering: theory and empirical test. Oikos 117(9): 1308-1320. DOI: 10.1111/j.0030$\underline{1299.2008 .16724 . x}$

Janzen DH. 1970. Herbivores and the number of tree species in tropical forests. The American Naturalist 104(940): 501-528.
Korndörfer CL, LR Dillenburg, LDS Duarte. 2015. Assessing the potential of Araucaria angustifolia (Araucariaceae) as a nurse plant in highland grasslands of south Brazil. New Zealand Journal of Botany 53(1): 5-14. DOI: 10.1080/0028825X.2014.979837

Korning J, H Balslev. 1994. Growth and mortality of trees in Amazonian tropical rain forest in Ecuador. Journal of Vegetation Science 5(1): 77-86. DOI 10.2307/3235641

Larson AJ, JA Lutz, DC Donato, JA Freund, ME Swanson, J HilleRisLambers, DG Sprugel, JF Franklin. 2015. Spatial aspects of tree mortality strongly differ between young and old-growth forests. Ecology 96(11): 2855-2861. DOI: $\underline{10.1890 / 15-0628.1}$

Liebsch D, MC Marques, R Goldenberg. 2008. How long does the Atlantic Rain Forest take to recover after a disturbance? Changes in species composition and ecological features during secondary succession. Biological Conservation 141(6): 1717-1725. DOI: 10.1016/j.biocon.2008.04.013

Marcon AK, AC Silva, P. Higuchi, TS Ferreira, FF Missio, B Salami, A Dalla Rosa, M Negrini, MA Bento, F Buzzi-Junior. 2014. Variação florístico-estrutural em resposta à heterogeneidade ambiental em uma floresta nebular em Ububici, Planalto Catarinense. Scientia Forestalis 42(103): 439-450.

Myers N, RA Mittermeier, CG Mittermeier, GAB Fonseca, J Kent. 2000. Biodiversity hotspots for conservation priorities. Nature 403: 853-858. DOI: 10.1038/35002501

Nascimento HEM, AS Dias, AAJ Tabanez, VM Viana. 1999. Estrutura e dinâmica de populações arbóreas de um fragmento de floresta estacional semidecidual na região de Piracicaba, SP. Revista Brasileira de Biologia 59(2): 329-342. DOI: 10.1590/S0034-71081999000200015

Nathan R, HC Muller-Landau. 2000. Spatial patterns of seed dispersal, their determinants and consequences for recruitment. Trends in Ecology \& Evolution 15(7): 278-285. DOI: $10.1016 / \mathrm{S} 0169-5347(00) 01874-7$

Ponce-Reyes R, E Nicholson, PW Baxter, RA Fuller, H Possingham. 2013. Extinction risk in cloud forest fragments under climate change and habitat loss. Diversity and Distributions 19(5-6): 518-529. DOI: 10.1111/ddi.12064

R Development Core Team. 2017. R: A language and environment for statistical computing. R Foundation for Statistical Computing. Consultado 20 may. 2017. Disponible en http:// www.r-project.org/.

Ripley BD. 1977. Modelling spatial patterns. Journal of the Royal Statistical Society. Series B (Methodological) 39(2): 172-212.

Salami B, P Higuchi, AC Silva, TS Ferreira, AK Marcon, F Buzzi Junior, MA Bento. 2014. Influência de variáveis ambientais na dinâmica do componente arbóreo em um fragmento de Floresta Ombrófila Mista em Lages, SC. Scientia Forestalis 42(102):197-207.

Sanquetta CR, AP Dalla Côrte, RL Eisfeld. 2003. Crescimento, mortalidade e recrutamento em duas florestas de Araucária (Araucaria angustifolia (Bert.) O. Ktze.) no Estado do Paraná, Brasil. Revista Ciências Exatas e Naturais 5(1): 101-112.

Sevegnani L, TC Silva, AL Gasper, L Meyer, M Verdi. 2012. Flora arbórea e o impacto humano nos fragmentos florestais na bacia do rio Pelotas, Santa Catarina, Brasil. Revista de Estudos Ambientais 14: 60-73. DOI: $10.7867 / 1983-1501.2012 \mathrm{v} 1$ $\underline{4 n 1 p 60-73}$

Sheil D, S Jennings, P Savill. 2000. Long-term permanent plot 
observations of vegetation dynamics in Budongo, a Ugandan rain forest. Journal of Tropical Ecology 16(6): 865882. DOI: $10.1017 / \mathrm{S} 0266467400001723$

Silveira AP, FR Martins, BS Menezes, FS Araujo. 2017. Is the spatial pattern of a tree population in a seasonally dry tropical climate explained by density-dependent mortality? Austral Ecology 43:191-202. DOI: 10.1111/aec.12556

Soboleski VF, P Higuchi, AC Silva, R Loebens, K Souza, F Buzzi Junior, CL Lima, AP Cruz, MAF Silva, JP Dallabrida. 2017. Variação de atributos funcionais do componente arbóreo em função de gradientes edáficos em uma floresta nebular no sul do Brasil. Rodriguesia 68: 291-300. DOI: $\underline{10.1590 / 2175-7860201768201}$

Sturgers HA. 1926. The choice of a class interval. Journal of the American Statistical Association 21(153): 65-66. DOI 10.1080/01621459.1926.10502161

Van Breugel M, M Martínez-Ramos, F Bongers. 2006. Community dynamics during early secondary succession in Mexican tropical rain forests. Journal of Tropical Ecology 22(6): 663-674. DOI: 10.1017/S0266467406003452

Recibido: $22 / 01 / 18$

Aceptado: 10/05/19 
